\title{
THE WEBSTER EQUATION REVISITED ${ }^{1}$
}

\author{
Sjoerd W. Rienstra \\ Department of Mathematics and Computing Science, \\ Eindhoven University of Technology, \\ The Netherlands, \\ s.w.rienstra@tue.nl
}

\begin{abstract}
The problem of low-frequency sound propagation in slowly varying ducts is systematically analysed as a perturbation problem of slow variation. The Webster equation and some variants are derived, and the entrance/exit plane boundary layer is given. It is shown why a varying lined duct in general does not have a solution.
\end{abstract}

\section{Introduction}

Sound of long wavelength, propagating in ducts of varying diameter like horns, is suitably described by an approximate equation, known as Webster's horn equation. This is an ordinary differential equation in the axial co-ordinate, and therefore forms a significant simplification of the problem. The extension of the multiple scales theory of sound propagation in slowly varying ducts $[1,2]$ naturally leads to this Webster equation. We found, however, no systematic derivation of this equation and its variants from the basic principles of perturbation methods.

The usual derivation is based on the assumption of a crosswise uniform acoustic pressure field, such that by averaging over a duct cross section the spatial dimensions of the problem are reduced from three to one.

Although it shows a remarkable evidence of ingenuity and deep physical insight, this derivation is mathematically not always satisfying. It is not clear (i) what exactly is the small parameter underlying the approximation, (ii) why the pressure may be assumed to be uniform, (iii) what the error is of the approximation, (iv) what the conditions are on the duct geometry and on the frequency of the field, (v) how to generalize to similar problems, (vi) how to generate higher order corrections, and (vii) what happens near the source or duct entrance or exit plane.

The practical importance of this equation justifies a more systematic approach, making precise under what conditions the theory is valid, and at the same time showing the way to generalise this equation.

We will consider various cases in detail. First, we show how a systematic approach, known as the method of slow variation, leads to the classic Webster's equation for hard-walled ducts.

By itself, the solution of Webster's equation is not a complete approximation of the prevailing equations in the duct. Actually, it is the outer solution of a non-uniform asymptotic expansion of the sound field. Near a source, or an entrance or exit plane, the field has, in terms of the small parameter, axially a boundary layer, a description of which will be given by an eigenfunction expansion.

Curved ducts, with a curvature radius of no more than the typical length scale of diameter variation, produce still the same equation.

The same type of analysis can be applied to ducts with lined walls. It is found that at any cross section, there are only non-trivial solutions possible for certain, geometry dependent, values of the wall impedance. As these impedance values vary along the duct, there are in general no solutions possible for the full duct.

\footnotetext{
${ }^{1}$ Presented at the 8th AIAA/CEAS Aeroacoustics Conference in Breckenridge, Colorado, 17-19 June 2002, paper nr. AIAA 20022520
} 
We continue with more general analyses of the problem of low frequency sound in a stagnant medium with slowly varying sound speed, and of sound in an irrotational isentropic mean flow, leading to generalised forms of Webster's equation.

\section{The physical models}

\subsection{The equations}

In the acoustic realm of a perfect gas that we will consider, we have for pressure $\tilde{p}$, velocity $\tilde{\boldsymbol{v}}$, density $\tilde{\rho}$, entropy $\tilde{s}$, and soundspeed $\tilde{c}$

$$
\begin{gathered}
\frac{\mathrm{d}}{\mathrm{d} t} \tilde{\rho}=-\tilde{\rho} \nabla \cdot \tilde{\boldsymbol{v}}, \quad \tilde{\rho} \frac{\mathrm{d}}{\mathrm{d} t} \tilde{\boldsymbol{v}}=-\nabla \tilde{p}, \quad \frac{\mathrm{d}}{\mathrm{d} t} \tilde{s}=0, \\
\tilde{s}=C_{V} \log \tilde{p}-C_{P} \log \tilde{\rho}, \tilde{c}^{2}=\frac{\gamma \tilde{p}}{\tilde{\rho}}, \gamma=\frac{C_{P}}{C_{V}} .
\end{gathered}
$$

where $\gamma, C_{P}$ and $C_{V}$ are gas constants. When we have a stationary mean flow with instationary timeharmonic perturbations of frequency $\omega$, given, in the usual complex notation, by

$$
\tilde{\boldsymbol{v}}=\boldsymbol{V}+\operatorname{Re}\left(\boldsymbol{v} \mathrm{e}^{\mathrm{i} \omega t}\right), \tilde{p}=P+\operatorname{Re}\left(p \mathrm{e}^{\mathrm{i} \omega t}\right), \tilde{\rho}=D+\operatorname{Re}\left(\rho \mathrm{e}^{\mathrm{i} \omega t}\right), \tilde{s}=S+\operatorname{Re}\left(s \mathrm{e}^{\mathrm{i} \omega t}\right),
$$

$(\omega>0)$ and linearize for small amplitude, we obtain for the mean flow

$$
\begin{gathered}
\nabla \cdot(D \boldsymbol{V})=0, \quad D(\boldsymbol{V} \cdot \nabla) \boldsymbol{V}=-\nabla P, \\
(\boldsymbol{V} \cdot \nabla) S=0, \quad S=C_{V} \log P-C_{P} \log D, \quad C^{2}=\frac{\gamma P}{D}
\end{gathered}
$$

and the perturbations

$$
\begin{gathered}
\mathrm{i} \omega \rho+\nabla \cdot(\boldsymbol{V} \rho+\boldsymbol{v} D)=0 \\
D(\mathrm{i} \omega+\boldsymbol{V} \cdot \nabla) \boldsymbol{v}+D(\boldsymbol{v} \cdot \nabla) \boldsymbol{V}+\rho(\boldsymbol{V} \cdot \nabla) \boldsymbol{V}=-\nabla p \\
(\mathrm{i} \omega+\boldsymbol{V} \cdot \nabla) s+\boldsymbol{v} \cdot \nabla S=0
\end{gathered}
$$

while

$$
s=\frac{C_{V}}{P} p-\frac{C_{P}}{D} \rho=\frac{C_{V}}{P}\left(p-C^{2} \rho\right) .
$$

Without mean flow, such that $V=\nabla P=0$, the equations may be reduced to (section 7)

$$
\nabla \cdot\left(C^{2} \nabla p\right)+\omega^{2} p=0
$$

If, in addition, the ambient medium is uniform, with a constant soundspeed $C$ and density $D$, the acoustic field becomes isentropic and irrotational and we may introduce a potential $\boldsymbol{v}=\nabla \phi$. Furthermore, equation (5) reduces to the Helmholtz equation. After introducing the free field wave number $k=\omega / C$ we have (sections $3,4,5,6$ )

$$
\nabla^{2} \phi+k^{2} \phi=0 .
$$

If the original flow field $\tilde{\boldsymbol{v}}$ is irrotational and isentropic everywhere (homentropic), we can introduce a potential for the velocity, where $\tilde{\boldsymbol{v}}=\nabla \tilde{\phi}$, and express $\tilde{p}$ as a function of $\tilde{\rho}$ only, such that we can integrate the momentum equation (Bernoulli's law, with constant $E$ ), to obtain for the mean flow

$$
\frac{1}{2} V^{2}+\frac{C^{2}}{\gamma-1}=E, \quad \nabla \cdot(D \boldsymbol{V})=0, \quad \frac{P}{D^{\gamma}}=\text { constant }
$$


and for the acoustic perturbations

$$
(\mathrm{i} \omega+\boldsymbol{V} \cdot \nabla) \rho+\rho \nabla \cdot \boldsymbol{V}+\nabla \cdot(D \nabla \phi)=0, \quad D(\mathrm{i} \omega+\boldsymbol{V} \cdot \nabla) \phi+p=0, \quad p=C^{2} \rho .
$$

These last equations are further simplified (eliminate $p$ and $\rho$ and use the fact that $\nabla \cdot(D \boldsymbol{V})=0$ ) to the rather general convected wave equation (section 8)

$$
D^{-1} \nabla \cdot(D \nabla \phi)-(\mathrm{i} \omega+\boldsymbol{V} \cdot \nabla)\left[C^{-2}(\mathrm{i} \omega+\boldsymbol{V} \cdot \nabla) \phi\right]=0
$$

\subsection{Nondimensionalisation}

Without further change of notation, we will assume throughout this paper that the problem is made dimensionless: lengths on a typical duct radius, time on typical sound speed / typical duct radius, etc.

\subsection{The geometry}

The domain of interest consists of a duct $\mathcal{V}$ of arbitrary cross section, slowly varying in axial direction (see figure 1).

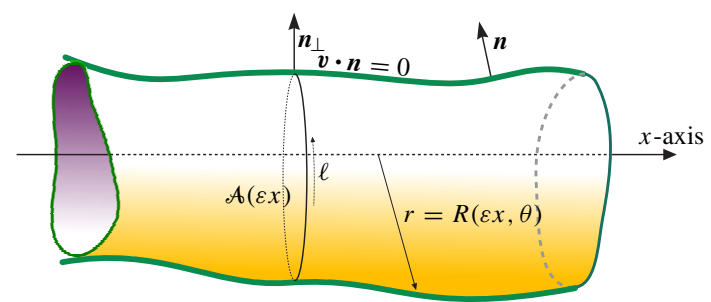

Figure 1. Sketch of geometry

For definiteness, it is given by the function $S$ in cylindrical coordinates as follows

$$
S(X, r, \theta)=r-R(X, \theta) \leq 0
$$

where $X=\varepsilon x \geqslant 0$ is a so-called slow variable while $\varepsilon$ is small. A cross section $\mathcal{A}(X)$ at $X$ has surface area $A(X)$. Whenever relevant ${ }^{2}$, we assume lengths made dimensionless such that

$$
A(0)=1 .
$$

At the duct surface $S=0$ the gradient $\nabla S$ is a vector normal to the surface (i.e., $\nabla S \propto \boldsymbol{n}$ ), while the transverse gradient $\nabla_{\perp} S$

$$
\nabla_{\perp}=\boldsymbol{e}_{r} \frac{\partial}{\partial r}+\boldsymbol{e}_{\theta} \frac{1}{r} \frac{\partial}{\partial \theta}, \text { with } \nabla_{\perp} S=\boldsymbol{e}_{r}-\boldsymbol{e}_{\theta} \frac{1}{r} R_{\theta}
$$

(where an index denotes a partial derivative) is directed in the plane of a cross section $\mathcal{A}(X)$, and normal to the duct circumference $\partial \mathcal{A}$. So if $\boldsymbol{n}_{\perp}$ is the component of the surface normal vector $\boldsymbol{n}$ in the plane of a cross section, we have $\nabla_{\perp} S \propto \boldsymbol{n}_{\perp}$.

\subsection{Frequency}

The frequencies considered are low, such that the corresponding typical wave number is of the same order of magnitude as the length scale of the duct variations, i.e., dimensionless $\mathcal{O}\left(\varepsilon^{-1}\right)$. In order to quantify this, we will rescale $k=\varepsilon \kappa$ and $\omega=\varepsilon \Omega$.

\footnotetext{
2 in particular in section 4
} 


\section{The classical problem}

\subsection{Equations and boundary conditions.}

The duct is semi-infinite and hard-walled. The solution is determined by a source at entrance plane $x=0$, and radiation conditions for $x \rightarrow \infty$. Other conditions, like a reflecting impedance plane at some exit plane $x=L$ (e.g., modelling a radiating open end), are also possible but they do not essentially alter the present analysis.

Inside $\mathcal{V}$ we have for acoustic potential $\phi$ (eq. 6)

$$
\nabla^{2} \phi+\varepsilon^{2} \kappa^{2} \phi=0 \text { if } \boldsymbol{x} \in \mathcal{V} \text {, with } \nabla \phi \cdot \boldsymbol{n}=0 \text { at } \boldsymbol{x} \in \partial \mathcal{V} .
$$

At the entrance interface $x=0$ we have a suitable boundary condition, say,

$$
\phi(0, r, \theta)=F(r, \theta) .
$$

The boundary condition of hard walls at $r=R(X, \theta)$ is given by

$$
\nabla_{\perp} \phi \cdot \nabla_{\perp} S=\phi_{r}-\frac{R_{\theta}}{R^{2}} \phi_{\theta}=\varepsilon R_{X} \phi_{x}
$$

Except for the immediate neighbourhood of the entrance plane, the typical axial variations of the acoustic field scale on the slow variable $X$, so we rewrite the equations and boundary conditions

$$
\varepsilon^{2} \phi_{X X}+\nabla_{\perp}^{2} \phi+\varepsilon^{2} \kappa^{2} \phi=0 \text {, with } \nabla \phi \cdot \nabla S=-\varepsilon^{2} \phi_{X} R_{X}+\nabla_{\perp} \phi \cdot \nabla_{\perp} S=0 \text { at } r=R .
$$

This rewriting in a slow variable is known as the method of slow variation[3]. Note that this equation has a small parameter multiplied with the highest derivative in $X$-direction, suggesting a singular perturbation problem $[4,5,6]$ with boundary layers in $X$.

\subsection{Asymptotic analysis: outer solution.}

Based on the observation that $\varepsilon^{2}$ is the only small parameter that occurs, we might be tempted to expand the solution in a Poincaré asymptotic power series in $\varepsilon^{2}$. However, this will be shown to be not exactly true. Depending on the behaviour of the solution near the entrance, the correction term should in general be $\mathcal{O}(\varepsilon)$ for matching. The leading and first order equations, however, will be equivalent. With the assumed Poincaré expansion of $\phi$, expressed in $X$,

$$
\phi(X, r, \theta ; \varepsilon)=\phi_{0}(X, r, \theta)+\varepsilon \phi_{1}(X, r, \theta)+\varepsilon^{2} \phi_{1}(X, r, \theta)+\ldots
$$

we obtain to leading order

$$
\nabla_{\perp}^{2} \phi_{0}=0, \text { with } \nabla_{\perp} \phi_{0} \cdot \boldsymbol{n}_{\perp}=0
$$

with solution $\phi_{0}=$ a constant. So $\phi_{0}=\phi_{0}(X)$, a function to be determined. To first order we have

$$
\nabla_{\perp}^{2} \phi_{1}=0, \text { with } \nabla_{\perp} \phi_{1} \cdot \boldsymbol{n}_{\perp}=0
$$

also with a constant solution, and so $\phi_{1}=\phi_{1}(X)$, a function to be determined. To second order we now have

$$
\nabla_{\perp}^{2} \phi_{2}+\phi_{0, X X}+\kappa^{2} \phi_{0}=0, \quad \text { with } \nabla_{\perp} \phi_{2} \cdot \boldsymbol{n}_{\perp}=\phi_{0, X} \frac{R R_{X}}{\sqrt{R^{2}+R_{\theta}^{2}}}
$$

The assumption (16) that there exists a Poincaré expansion for $\phi$, expressed in this slow variable $X$, is not trivial (Poincaré expansions are critically dependent of the variables chosen!). It requires certain solvability conditions for, e.g., $\phi_{2}$, yielding an equation for $\phi_{0}$. To obtain this, we integrate along a cross section $\mathcal{A}(X)$ and apply Gauss' theorem

$$
\iint_{\mathcal{A}} \nabla_{\perp}^{2} \phi_{2} \mathrm{~d} \sigma=\int_{\partial \mathcal{A}} \nabla_{\perp} \phi_{2} \cdot \boldsymbol{n}_{\perp} \mathrm{d} \ell=\int_{\partial \mathcal{A}} \phi_{0, X} \frac{R R_{X}}{\sqrt{R^{2}+R_{\theta}^{2}}} \mathrm{~d} \ell=\ldots
$$


Then we parametrize $\partial \mathcal{A}$ with $\theta$, such that $\mathrm{d} \ell=\sqrt{R^{2}+R_{\theta}^{2}} \mathrm{~d} \theta$, and we continue

$$
=\int_{0}^{2 \pi} \phi_{0, X} R R_{X} \mathrm{~d} \theta=\phi_{0, X} \int_{0}^{2 \pi} R R_{X} \mathrm{~d} \theta=\phi_{0, X} A_{X} .
$$

On the other hand, we also have

$$
\iint_{\mathcal{A}}\left[\phi_{0, X X}+\kappa^{2} \phi_{0}\right] \mathrm{d} \sigma=A\left(\phi_{0, X X}+\kappa^{2} \phi_{0}\right)
$$

Altogether we have for $\phi_{0}$ the equation

$$
A^{-1}\left(A \phi_{0, X}\right)_{X}+\kappa^{2} \phi_{0}=0
$$

which is indeed Webster's equation[7, 8] in properly scaled coordinates.

Evidently, the first order solution follows the same pattern and satisfies also

$$
A^{-1}\left(A \phi_{1, X}\right)_{X}+\kappa^{2} \phi_{1}=0
$$

\subsection{Solutions of Webster's equation.}

Webster's equation can be recast into a more transparent form by the transformation[9, 10, 11, 12, 13]

$$
A(X)=d(X)^{2}, \quad \phi=d^{-1} \psi,
$$

leading to

$$
\psi^{\prime \prime}+\left(\kappa^{2}-\frac{d^{\prime \prime}}{d}\right) \psi=0
$$

Depending on the sign of $\kappa^{2}-d^{\prime \prime} / d$, the solutions behave like propagating or exponentially decaying waves. Exponential or sinusoidal solutions are readily found for geometries with $d^{\prime \prime} / d=m^{2}$, a constant, yielding Salmon's family of horns $[9,10]$

$$
d(X)=a \mathrm{e}^{m X}+b \mathrm{e}^{-m X}
$$

where $a, b$, and $m$ are constants. If $m \rightarrow 0$ such that $a=\frac{1}{2}\left(A_{0}+A_{1} / m\right)$ and $b=\frac{1}{2}\left(A_{0}-A_{1} / m\right)$, the shape reduces to the conical horn $d(X)=A_{0}+A_{1} X$. For $b=0$ we have the exponential horn, and if $b=a$ the catenoidal horn. The parameter $m$ is clearly most important since it determines whether the wave is propagating $(m<\kappa)$ or cut-off $(m>\kappa)$.

\subsection{Boundary conditions in $X$.}

The above equation for $\phi_{0}$ and $\phi_{1}$ is of second order and therefore two boundary conditions are required to determine the solution. For $X \rightarrow \infty$ we have the condition of radiation. At $X=0, \phi_{0}$ and $\phi_{1}$ cannot satisfy the $(r, \theta)$-dependent boundary condition (13). Indeed, as anticipated before, near $x=0$ there is a boundary layer of $X=\mathcal{O}(\varepsilon)$, i.e., $x=\mathcal{O}(1)$, which determines the (outer) solutions $\phi_{0}$ and $\phi_{1}$ via conditions of matching. This will be considered in the next section.

\section{Entrance boundary layer}

Near the entrance, for $X=\mathcal{O}(\varepsilon)$, i.e., $x=\mathcal{O}(1)$, we have of course equation (12)

$$
\nabla^{2} \phi+\varepsilon^{2} \kappa^{2} \phi=0 \text { if } \boldsymbol{x} \in \mathcal{V}, \quad \text { with } \nabla_{\perp} \phi \cdot \boldsymbol{n}=0 \text { at } \boldsymbol{x} \in \partial \mathcal{V} .
$$

Up to $\mathcal{O}\left(\varepsilon^{2}\right)$, this Helmholtz equation is equivalent to the Laplace equation. Therefore, the boundary layer analysis is essentially similar to the one for the heat equation, discussed in Chandra[14]. Expand

$$
\phi(X, r, \theta ; \varepsilon)=\Phi_{0}(x, r, \theta)+\varepsilon \Phi_{1}(x, r, \theta)+\mathcal{O}\left(\varepsilon^{2}\right)
$$


so we have inside $\mathcal{V}$ to leading and first order

$$
\begin{array}{ll}
\mathcal{O}(1): & \nabla^{2} \Phi_{0}=0, \\
\mathcal{O}(\varepsilon): & \nabla^{2} \Phi_{1}=0 .
\end{array}
$$

At $x=0$ we have from (13) the initial conditions

$$
\Phi_{0}(0, r, \theta)=F(r, \theta), \quad \Phi_{1}(0, r, \theta)=0
$$

For $x \rightarrow \infty$ conditions of matching with the outer solution $\phi_{0}+\varepsilon \phi_{1}$ apply. For the boundary condition at $r=R$ we have to expand $R(\varepsilon x, \theta)$. Note that for any function $f$

$$
f(R(\varepsilon x) ; \varepsilon)=f\left(R+\varepsilon x R_{X}+\mathcal{O}\left(\varepsilon^{2}\right) ; \varepsilon\right)=f_{0}(R)+\varepsilon\left(f_{1}(R)+x f_{0, r}(R) R_{X}\right)+\mathcal{O}\left(\varepsilon^{2}\right)
$$

where $R$ without any argument denotes the value at $X=0$. Furthermore, we have

$$
\frac{R_{\theta}(X, \theta)}{R^{2}(X, \theta)}=\frac{R_{\theta}}{R^{2}}+\varepsilon x\left(\frac{R_{X}}{R^{2}}\right)_{\theta}+\mathcal{O}\left(\varepsilon^{2}\right)
$$

So at the boundary

$$
\begin{aligned}
\nabla_{\perp} \phi \cdot \nabla_{\perp} S=\phi_{r}-\frac{R_{\theta}}{R^{2}} \phi_{\theta}=\Phi_{0, r} & -\frac{R_{\theta}}{R^{2}} \Phi_{0, \theta}+\varepsilon\left[\Phi_{1, r}-\frac{R_{\theta}}{R^{2}} \Phi_{1, \theta}\right. \\
& \left.+x \Phi_{0, r r} R_{X}-x \frac{R_{\theta}}{R^{2}} R_{X} \Phi_{0, r \theta}-x\left(\frac{R_{X}}{R^{2}}\right)_{\theta} \Phi_{0, \theta}\right]=\varepsilon R_{X} \Phi_{0, x}
\end{aligned}
$$

which means at $r=R(0, \theta)$ for the leading and first order

$$
\begin{aligned}
& \nabla_{\perp} \Phi_{0} \cdot \nabla_{\perp} S_{0}=\Phi_{0, r}-\frac{R_{\theta}}{R^{2}} \Phi_{0, \theta}=0 \\
& \nabla_{\perp} \Phi_{1} \cdot \nabla_{\perp} S_{0}=\Phi_{1, r}-\frac{R_{\theta}}{R^{2}} \Phi_{1, \theta}=R_{X} \Phi_{0, x}-x \Phi_{0, r r} R_{X}+x \frac{R_{\theta}}{R^{2}} R_{X} \Phi_{0, r \theta}+x\left(\frac{R_{X}}{R^{2}}\right)_{\theta} \Phi_{0, \theta},
\end{aligned}
$$

where $S_{0}=S(0, r, \theta)$.

It is important for the subsequent matching to note that the solutions of (28) with (33) are only defined up to a linear term $K x$. For $\Phi_{0}$, however, this would result in terms of $\mathcal{O}\left(\varepsilon^{-1}\right)$ if $x=\mathcal{O}\left(\varepsilon^{-1}\right)$ which do not match with an outer solution $\phi_{0}=\mathcal{O}(1)$. Therefore, we will not include this extra term. For $\Phi_{1}$, on the other hand, we will have to retain the possibility, and in the end a linear term $K_{1} x$ will be added, where $K_{1}$ must be determined by the matching.

From the identity at $r=R$

$$
\frac{\mathrm{d}}{\mathrm{d} \theta} \Phi_{0, \theta}=\Phi_{0, r \theta} R_{\theta}+\Phi_{0, \theta \theta}
$$

and with the defining equation applied at $r=R$ while using relation (33a)

$$
-\Phi_{0, r r}=\frac{1}{R} \Phi_{0, r}+\frac{1}{R^{2}} \Phi_{0, \theta \theta}+\Phi_{0, x x}=\frac{R_{\theta}}{R^{3}} \Phi_{0, \theta}+\frac{1}{R^{2}} \Phi_{0, \theta \theta}+\Phi_{0, x x}
$$

it follows that equation (33b) is equivalent to

$$
\nabla_{\perp} \Phi_{1} \cdot \nabla_{\perp} S_{0}=\left.Q_{0}(x, \theta) \stackrel{\text { def }}{=} R_{X} \Phi_{0, x}\right|_{r=R}+\frac{x}{R}\left\{\left.R R_{X} \Phi_{0, x x}\right|_{r=R}+\frac{\mathrm{d}}{\mathrm{d} \theta}\left(\left.\frac{R_{X}}{R} \Phi_{0, \theta}\right|_{r=R}\right)\right\}
$$




\subsection{Leading order.}

The right-running solution $\Phi_{0}$ (only non-increasing exponentials are allowed for matching) may be expressed by the eigenfunction expansion

$$
\Phi_{0}(\boldsymbol{x})=\sum_{n=0}^{\infty} F_{n} \psi_{n}(r, \theta) \mathrm{e}^{-\lambda_{n} x}
$$

where

$$
\nabla_{\perp}^{2} \psi_{n}+\lambda_{n}^{2} \psi_{n}=0, \quad \nabla_{\perp} \psi_{n} \cdot \nabla_{\perp} S_{0}=0
$$

with $\lambda_{0}=0, \psi_{0}$ is a constant (normalised to 1 ), the other eigenvalues $\lambda_{n}$ are real positive, and the eigenfunctions $\psi_{n}$ are real, orthogonal and assumed normalized. In general these eigenfunctions are to be determined numerically. However, if the duct is cylindrical (i.e., $R$ is independent of $\theta$ ), we have

$$
\psi_{n}(r, \theta)=N_{n} J_{v}\left(j_{v \mu}^{\prime} r / R\right) \mathrm{e}^{ \pm \mathrm{i} v \theta}, \quad v \in \mathbb{N}
$$

where $J_{v}$ is the $\nu$-th order ordinary Bessel function of the 1st kind[15], and $j_{v \mu}^{\prime}$ is the $\mu$-th (real-valued, positive) zero of $J_{v}^{\prime}$, ordered such that they are monotonically increasing. $N_{n}$ is a normalisation constant, and the corresponding eigenvalue is of course $\lambda_{n}=j_{v \mu}^{\prime} / R$.

The amplitudes are determined from the entrance interface $x=0$ as follows

$$
F_{n}=\iint_{\mathcal{A}(0)} F(r, \theta) \psi_{n}(r, \theta) \mathrm{d} \sigma .
$$

Note that, as $\psi_{n}$ are orthonormal, the axial flux is to leading order proportional to the imaginary part of

$$
\int_{0}^{2 \pi} \int_{0}^{R} \Phi_{0} \Phi_{0, x}^{*} r \mathrm{~d} r \mathrm{~d} \theta=-\sum_{n=1}^{\infty} \lambda_{n}\left|F_{n}\right|^{2} \mathrm{e}^{-2 \lambda_{n} x} .
$$

As this expression is real, its imaginary part is zero and, thus, the axial flux vanishes to leading order. Indeed, the outer solution is a slowly varying function of $X$ and therefore the flux, proportional to the axial derivative, is $\mathcal{O}(\varepsilon)$.

For $x \rightarrow \infty$, the exponential terms in $\Phi_{0}(x)$ vanish and we have

$$
\Phi_{0}(\boldsymbol{x}) \simeq F_{0} .
$$

\subsection{1st Order.}

$\mathcal{Q}_{0}$, the right hand side of equation (36), may be written as

$$
\begin{aligned}
\mathcal{Q}_{0}(x, \theta) & =\sum_{n=1}^{\infty} F_{n} \mathrm{e}^{-\lambda_{n} x}\left[-\left.R_{X} \lambda_{n} \psi_{n}\right|_{r=R}+\left.x R_{X} \lambda_{n}^{2} \psi_{n}\right|_{r=R}+\frac{x}{R} \frac{\mathrm{d}}{\mathrm{d} \theta}\left(\left.\frac{R_{X}}{R} \psi_{n, \theta}\right|_{r=R}\right)\right] \\
& =R^{-1} \sum_{n=1}^{\infty} F_{n}\left[-\left.\lambda_{n} R R_{X}\left(x \mathrm{e}^{-\lambda_{n} x}\right)_{x} \psi_{n}\right|_{r=R}+x \mathrm{e}^{-\lambda_{n} x} \frac{\mathrm{d}}{\mathrm{d} \theta}\left(\left.\frac{R_{X}}{R} \psi_{n, \theta}\right|_{r=R}\right)\right]
\end{aligned}
$$

To solve the problem for $\Phi_{1}$, we introduce a Green's function $G(\boldsymbol{x} ; \boldsymbol{\xi})$ with $\boldsymbol{x}=(x, r, \theta)$ and $\boldsymbol{\xi}=(\xi, \rho, \eta)$ satisfying

$$
\begin{gathered}
\nabla_{\perp}^{2} G+\frac{\partial^{2}}{\partial x^{2}} G=-\delta(\boldsymbol{x}-\boldsymbol{\xi}), \\
\frac{\partial}{\partial n} G=0 \text { at } r=R(0, \theta), \\
G(\boldsymbol{x} ; \boldsymbol{\xi})=0 \text { at } x=0 . \\
G(\boldsymbol{x} ; \boldsymbol{\xi}) \rightarrow \text { a constant }, x \rightarrow \infty . \\
x \frac{\partial}{\partial x} G(\boldsymbol{x} ; \boldsymbol{\xi}) \rightarrow 0, x \rightarrow \infty .
\end{gathered}
$$


We determine the Green's function by applying the Fourier Sine Transform ${ }^{3}$ with respect to $x(x \rightarrow \alpha)$ to (44), to obtain

$$
\nabla_{\perp}^{2} \hat{G}-\alpha^{2} \hat{G}=-\sqrt{\frac{2}{\pi}} \sin (\alpha \xi) \delta\left(x_{\perp}-\xi_{\perp}\right) .
$$

where $\boldsymbol{x}_{\perp}$ denotes the transverse component of $\boldsymbol{x}$, i.e., $\boldsymbol{x}_{\perp}=(r, \theta)$ (similarly for $\boldsymbol{\xi}_{\perp}$ ). We assume that the Green's function can be expanded by the same basis function as has been used for $\Phi_{0}$

$$
\hat{G}(\alpha, r, \theta ; \boldsymbol{\xi})=\sum_{m=0}^{\infty} a_{m}(\alpha, \xi) \psi_{m}(r, \theta)
$$

Therefore

$$
\nabla^{2} \hat{G}=-\sum_{m=0}^{\infty} a_{m} \lambda_{m}^{2} \psi_{m}(r, \theta)
$$

Substituting this into (45) yields

$$
\sum_{m=0}^{\infty} a_{m} \psi_{m}\left(\lambda_{m}^{2}+\alpha^{2}\right)=\sqrt{\frac{2}{\pi}} \sin (\alpha \xi) \delta\left(\boldsymbol{x}_{\perp}-\boldsymbol{\xi}_{\perp}\right) .
$$

Next, we multiply (46) with $\psi_{n}$ and integrate over the cross section $\mathcal{A}(0)$ to obtain

$$
\iint_{\mathcal{A}(0)} \sum_{m=0}^{\infty} a_{m} \psi_{n} \psi_{m}\left(\lambda_{m}^{2}+\alpha^{2}\right) \mathrm{d} \sigma=\sqrt{\frac{2}{\pi}} \iint_{\mathcal{A}(0)} \psi_{n}(r, \theta) \sin (\alpha \xi) \delta\left(\boldsymbol{x}_{\perp}-\boldsymbol{\xi}_{\perp}\right) \mathrm{d} \sigma .
$$

Orthonormality of the basis functions yields

$$
a_{m}=\sqrt{\frac{2}{\pi}} \frac{\sin (\alpha \xi)}{\lambda_{m}^{2}+\alpha^{2}} \psi_{m}(\rho, \eta) .
$$

Therefore,

$$
\hat{G}(\alpha, r, \theta ; \xi, \rho, \eta)=\sqrt{\frac{2}{\pi}} \sum_{m=0}^{\infty} \frac{\sin (\alpha \xi)}{\lambda_{m}^{2}+\alpha^{2}} \psi_{m}(\rho, \eta) \psi_{m}(r, \theta) .
$$

The inverse Fourier Sine Transform yields

$$
G(\boldsymbol{x} ; \boldsymbol{\xi})=\frac{2}{\pi} \sum_{m=0}^{\infty} \psi_{m}(\rho, \eta) \psi_{m}(r, \theta) \int_{0}^{\infty} \frac{\sin (\alpha x) \sin (\alpha \xi)}{\lambda_{m}^{2}+\alpha^{2}} \mathrm{~d} \alpha,
$$

where[15] for $\lambda_{0}=0$

$$
\int_{0}^{\infty} \frac{\sin (\alpha x) \sin (\alpha \xi)}{\alpha^{2}} \mathrm{~d} \alpha=\frac{1}{2} \pi \min (x, \xi)
$$

and for $\lambda_{m}>0$,

$$
\int_{0}^{\infty} \frac{\sin (\alpha x) \sin (\alpha \xi)}{\lambda_{m}^{2}+\alpha^{2}} \mathrm{~d} \alpha=\frac{1}{2} \pi \mathrm{e}^{-\lambda_{m} \max (x, \xi)} \frac{1}{\lambda_{m}} \sinh \left(\lambda_{m} \min (x, \xi)\right) .
$$

Therefore, the Green's function becomes

$$
\begin{aligned}
G(\boldsymbol{x} ; \boldsymbol{\xi}) & =x+\sum_{m=1}^{\infty} \psi_{m}(\rho, \eta) \psi_{m}(r, \theta) \mathrm{e}^{-\lambda_{m} \xi} \frac{\sinh \left(\lambda_{m} x\right)}{\lambda_{m}} \text { if } 0 \leq x \leq \xi, \\
& =\xi+\sum_{m=1}^{\infty} \psi_{m}(\rho, \eta) \psi_{m}(r, \theta) \mathrm{e}^{-\lambda_{m} x} \frac{\sinh \left(\lambda_{m} \xi\right)}{\lambda_{m}} \text { if } 0 \leq \xi \leq x .
\end{aligned}
$$

\footnotetext{
${ }^{3}$ where $\hat{f}(\alpha)=\sqrt{\frac{2}{\pi}} \int_{0}^{\infty} \sin (\alpha x) f(x) \mathrm{d} x, f(x)=\sqrt{\frac{2}{\pi}} \int_{0}^{\infty} \sin (\alpha x) \hat{f}(\alpha) \mathrm{d} \alpha$.
} 
Note that as $x \rightarrow \infty, G$ tends to $\xi$ and $\frac{\partial G}{\partial x}$ tends to zero exponentially.

Using this Green's function, we obtain for $\Phi_{1}$ the following relation, to be integrated over domain $\mathcal{V}$,

$$
\Phi_{1} \delta(\boldsymbol{x}-\boldsymbol{\xi})=G \nabla^{2} \Phi_{1}-\Phi_{1} \nabla^{2} G .
$$

However, since $\Phi_{1} \sim K_{1} \xi$ for large $\xi$ (see the remark below equations 33), this yields a divergent integral as the domain here is a semi-infinite duct. Therefore, we consider a region $\mathcal{V}^{\prime}$ with a finite length $0 \leq x \leq$ $x_{0}$, where $x_{0}$ is small compared to $\varepsilon^{-1}$, but large enough for all exponential terms to practically vanish. Integrate (54) along domain $\mathcal{V}^{\prime}$ and by using Green's second identity we get

$$
\begin{aligned}
\Phi_{1}(\boldsymbol{\xi})= & \iiint_{\mathcal{V}^{\prime}}\left(G \Delta \Phi_{1}-\Phi_{1} \Delta G\right) \mathrm{d} \boldsymbol{x}=\iint_{x=0}\left(-G \frac{\partial \Phi_{1}}{\partial x}+\Phi_{1} \frac{\partial G}{\partial x}\right) \mathrm{d} \sigma \\
& \quad+\iint_{r=R(0, \eta)}\left(G \nabla_{\perp} \Phi_{1}-\Phi_{1} \nabla_{\perp} G\right) \cdot \boldsymbol{n}_{\perp} \mathrm{d} \sigma+\iint_{x=x_{0}}\left(G \frac{\partial \Phi_{1}}{\partial x}-\Phi_{1} \frac{\partial G}{\partial x}\right) \mathrm{d} \sigma \\
= & \iint_{r=R(0, \eta)} \frac{G Q_{0}(x, \theta)}{\left|\nabla_{\perp} S\right|} \mathrm{d} \ell \mathrm{d} \xi+K_{1} \xi .
\end{aligned}
$$

Since $\left|\nabla_{\perp} S\right|=\frac{1}{R} \sqrt{R^{2}+R_{\theta}^{2}}$ and $\mathrm{d} \ell=\sqrt{R^{2}+R_{\theta}^{2}} \mathrm{~d} \theta$, we obtain

$$
\Phi_{1}(\boldsymbol{\xi})=\left.\int_{0}^{2 \pi} \int_{0}^{\infty} \mathcal{Q}_{0}(x, \theta) G(\boldsymbol{x} ; \boldsymbol{\xi})\right|_{r=R} R \mathrm{~d} x \mathrm{~d} \theta+K_{1} \xi
$$

As we have $\mathcal{Q}_{0}$ in the form of a series expansion, we can write

$$
\begin{aligned}
\Phi_{1}(\boldsymbol{\xi})=K_{1} \xi+\sum_{n=1}^{\infty} & F_{n} \int_{0}^{2 \pi}\left[-\left.\left.R R_{X} \lambda_{n} \psi_{n}\right|_{r=R} \int_{0}^{\infty} \mathrm{e}^{-\lambda_{n} x} G(\boldsymbol{x} ; \boldsymbol{\xi})\right|_{r=R} \mathrm{~d} x\right. \\
& \left.+\left.\left\{\left.R R_{X} \lambda_{n}^{2} \psi_{n}\right|_{r=R}+\frac{\mathrm{d}}{\mathrm{d} \theta}\left(\left.\frac{R_{X}}{R} \psi_{n, \theta}\right|_{r=R}\right)\right\} \int_{0}^{\infty} x \mathrm{e}^{-\lambda_{n} x} G(\boldsymbol{x} ; \boldsymbol{\xi})\right|_{r=R} \mathrm{~d} x\right] \mathrm{d} \theta
\end{aligned}
$$

It may be noted that the normal derivative of this series does not converge uniformly near the wall. For all basis functions, $\nabla_{\perp} \psi_{n} \cdot \boldsymbol{n}_{\perp}=0$, whereas $\nabla_{\perp} \Phi_{1} \cdot \boldsymbol{n}_{\perp} \neq 0$.

Finally, we remove the $x$-integration by

$$
\begin{aligned}
&\left.\int_{0}^{\infty} \mathrm{e}^{-\lambda_{n} x} G(\boldsymbol{x} ; \boldsymbol{\xi})\right|_{r=R} \mathrm{~d} x=\frac{1-\mathrm{e}^{-\lambda_{n} \xi}}{\lambda_{n}^{2}}-\sum_{m=1}^{\infty} \psi_{m}(R, \theta) \psi_{m}(\rho, \eta) \frac{\mathrm{e}^{-\lambda_{n} \xi}-\mathrm{e}^{-\lambda_{m} \xi}}{\lambda_{n}^{2}-\lambda_{m}^{2}}, \\
&\left.\int_{0}^{\infty} x \mathrm{e}^{-\lambda_{n} x} G(\boldsymbol{x} ; \boldsymbol{\xi})\right|_{r=R} \mathrm{~d} x=\frac{2-\left(2+\lambda_{n} \xi\right) \mathrm{e}^{-\lambda_{n} \xi}}{\lambda_{n}^{3}} \\
&-\sum_{m=1}^{\infty} \psi_{m}(R, \theta) \psi_{m}(\rho, \eta) \frac{2 \lambda_{n}\left(\mathrm{e}^{-\lambda_{n} \xi}-\mathrm{e}^{-\lambda_{m} \xi}\right)+\xi\left(\lambda_{n}^{2}-\lambda_{m}^{2}\right) \mathrm{e}^{-\lambda_{n} \xi}}{\left(\lambda_{n}^{2}-\lambda_{m}^{2}\right)^{2}} .
\end{aligned}
$$

If $m=n$, the limit $\lambda_{m} \rightarrow \lambda_{n}$ should be taken.

For $x \rightarrow \infty$, the exponential terms in $\Phi_{1}(\boldsymbol{x})$ vanish and we have (we exchange the variables $\boldsymbol{x}$ and $\boldsymbol{\xi}$ )

$$
\Phi_{1}(\boldsymbol{x}) \simeq K_{1} x+\sum_{n=1}^{\infty} F_{n} \int_{0}^{2 \pi}\left[\left.R R_{X} \lambda_{n}^{-1} \psi_{n}\right|_{\rho=R}+\frac{2}{\lambda_{n}} \frac{\mathrm{d}}{\mathrm{d} \eta}\left(\left.\frac{R_{X}}{R} \psi_{n, \eta}\right|_{\rho=R}\right)\right] \mathrm{d} \eta
$$

By using the periodicity of $\psi_{n}$ in its circumferential argument $\eta$, we have finally

$$
\Phi_{1}(\boldsymbol{x}) \simeq K_{1} x+\left.\sum_{n=1}^{\infty} \frac{F_{n}}{\lambda_{n}} \int_{0}^{2 \pi} R R_{X} \psi_{n}\right|_{\rho=R} \mathrm{~d} \eta
$$




\subsection{Matching.}

Both the initial conditions for $\phi_{0}$ and $\phi_{1}$ and the constant $K_{1}$ are determined from matching with the outer solution. From equations (42) and (60) we have

$$
\phi_{0}(0)+X \phi_{0, X}(0)+\varepsilon \phi_{1}(0) \sim F_{0}+\varepsilon K_{1} x+\left.\varepsilon \sum_{n=1}^{\infty} \frac{F_{n}}{\lambda_{n}} \int_{0}^{2 \pi} R R_{X} \psi_{n}\right|_{\rho=R} \mathrm{~d} \eta
$$

and so we find

$$
\left.\begin{array}{rl}
\phi_{0}(0) & =F_{0} \\
K_{1} & =\phi_{0, X}(0) \\
\phi_{1}(0) & =\left.\sum_{n=1}^{\infty} \frac{F_{n}}{\lambda_{n}} \int_{0}^{2 \pi} R R_{X} \psi_{n}\right|_{\rho=R} \mathrm{~d} \eta
\end{array}\right\}
$$

\section{Curved ducts}

The present results remain valid for the slightly more general problem of curved ducts (like certain musical instruments) if the curvature of the duct axis (and its derivative) is $\mathcal{O}(\varepsilon)$. Together with the assumed slow variation in the axial coordinate, the associated orthogonal coordinate system (based on the tangent and possibly- the normal and binormal of the curve that describes the duct axis) leave the Laplacian unchanged up to $\mathcal{O}\left(\varepsilon^{3}\right)$.

A simple example is the inside of a perturbed torus, described by a fixed torus radius $\varepsilon^{-1}$ and slowly varying tube radius $R$. With local (polar-type) coordinates $\xi, r, \varphi$, we define

$$
\begin{gathered}
x=\varepsilon^{-1}(1+\varepsilon r \cos \theta) \cos (\varepsilon \xi), \\
y=\varepsilon^{-1}(1+\varepsilon r \cos \theta) \sin (\varepsilon \xi), \quad z=r \sin \theta,
\end{gathered}
$$

where $0 \leq r \leq R(\varepsilon \xi, \theta), 0 \leq \theta<2 \pi, 0 \leq \varepsilon \xi<2 \pi$. If we write $X=\varepsilon \xi$, we get (cf. equation 6)

$$
\begin{aligned}
\nabla^{2} \phi & +\varepsilon^{2} \kappa^{2} \phi \\
& =\nabla_{\perp}^{2} \phi+\varepsilon^{2}(1+\varepsilon r \cos \theta)^{-2} \frac{\partial^{2}}{\partial X^{2}} \phi+\varepsilon(1+\varepsilon r \cos \theta)^{-1}\left[\cos \theta \frac{\partial}{\partial r} \phi-\frac{1}{r} \frac{\partial}{\partial \theta} \phi\right]+\varepsilon^{2} \kappa^{2} \phi=0 .
\end{aligned}
$$

Boundary conditions at $S=r-R(X, \theta)=0$ are

$$
\nabla_{\perp} \phi \cdot \nabla_{\perp} S-\frac{\varepsilon^{2} R_{X} \phi_{X}}{(1+\varepsilon r \cos \theta)^{2}}=0 .
$$

If we expand $\phi=\phi_{0}+\varepsilon \phi_{1}+\varepsilon^{2} \phi_{2}+\ldots$, we get to leading order

$$
\nabla_{\perp}^{2} \phi_{0}=0, \nabla_{\perp} \phi_{0} \cdot \boldsymbol{n}_{\perp}=0,
$$

so $\phi_{0}=\phi_{0}(X)$. Then $\frac{\partial}{\partial r} \phi_{0}=\frac{\partial}{\partial \theta} \phi_{0}=0$ and we have also

$$
\nabla_{\perp}^{2} \phi_{1}=0, \nabla_{\perp} \phi_{1} \cdot \boldsymbol{n}_{\perp}=0,
$$

leading to $\phi_{1}=\phi_{1}(X)$. So again $\frac{\partial}{\partial r} \phi_{1}=\frac{\partial}{\partial \theta} \phi_{1}=0$ and we obtain again

$$
\nabla_{\perp}^{2} \phi_{2}+\phi_{0, X X}+\kappa^{2} \phi_{0}=0, \quad \text { with } \nabla_{\perp} \phi_{2} \cdot \nabla_{\perp} S=\phi_{0, X} R_{X},
$$

yielding thus, after a similar argument as before, Webster's equation. 


\section{Impedance walls}

If the duct walls is equipped with an impedance-type acoustic lining, we will in general expect solutions, which will decay exponentially in axial direction. Therefore, in the compressed variable $X$, only trivial (i.e., zero) solutions will exist. We will see that this is by and large the case, although for a purely imaginary impedance in a straight duct there are exceptions.

The impedance-wall boundary condition at $r=R$ is given by

$$
\nabla \phi \cdot \boldsymbol{n}=-\frac{\mathrm{i} \varepsilon \kappa}{Z} \phi=\zeta \phi
$$

with specific impedance $Z$. As before, we assume the Poincaré expansion $\phi=\phi_{0}+\varepsilon \phi_{1}+\varepsilon^{2} \phi_{2}+\ldots$. First we note that it is easily verified, that if $Z=0$ only the trivial solutions $\phi_{0}=\phi_{1}=0$ occur. Then we consider two possibilities: $Z=\mathcal{O}(1)$ and $Z=\mathcal{O}(\varepsilon)$.

\section{1 $Z=\mathcal{O}(1)$}

As $\zeta=\mathcal{O}(\varepsilon)$, we write $\zeta=\varepsilon \zeta_{1}$. In this case we have only trivial solutions. Expand equations and boundary conditions as before, to get to leading order

$$
\nabla_{\perp}^{2} \phi_{0}=0 \text {, with } \nabla_{\perp} \phi_{0} \cdot \boldsymbol{n}_{\perp}=0
$$

with solution $\phi_{0}=\phi_{0}(X)$, a function to be determined. To first order we have

$$
\nabla_{\perp}^{2} \phi_{1}=0 \text {, with } \nabla_{\perp} \phi_{1} \cdot \boldsymbol{n}_{\perp}=\zeta_{1} \phi_{0} .
$$

Since

$$
\iint_{\mathcal{A}} \nabla_{\perp}^{2} \phi_{1} \mathrm{~d} \sigma=\zeta_{1} \phi_{0} \int_{\partial \mathcal{A}} \mathrm{d} \ell=0
$$

we must have $\phi_{0}=0$, and so $\phi_{1}=\phi_{1}(X)$. Nothing changes when we continue, and so all terms of the expansion vanish.

\section{2 $Z=\mathcal{O}(\varepsilon)$}

Now we have $\zeta=\mathcal{O}(1)$, which changes the boundary condition expansion. To leading order we have

$$
\nabla^{2} \phi_{0}=0 \text { in } \mathcal{A} \text {, with } \nabla \phi_{0} \cdot \boldsymbol{n}=\zeta \phi_{0} \text { at } \partial \mathcal{A} \text {. }
$$

This is an eigenvalue problem for the Dirichlet-to-Neumann operator[14] $\mathcal{N}: f \mapsto g$, that maps a given Dirichlet boundary value $f$ to the normal derivative $g$ of $f$ 's harmonic extension into $\mathcal{A}$. In other words, $\mathcal{N}(f)=\left.\frac{\partial}{\partial n} \psi\right|_{\partial \mathcal{A}}$ where $\psi$ is the solution of

$$
\nabla^{2} \psi=0 \text { in } \mathcal{A} \text {, with } \psi=f \text { at } \partial \mathcal{A} \text {. }
$$

As we are looking for $\mathcal{N}\left(\phi_{0}\right)=\zeta \phi_{0}$, equation (72) corresponds to the eigenvalue problem of $\mathcal{N}$.

From Green's 2nd identity, applied to $\phi_{0}$ and its complex conjugate, it can be deduced that any possible $\zeta$ is real. Furthermore, from Green's 1st identity applied to $\phi_{0}$ it follows that any possible $\zeta$ is positive, and $Z$ is thus negative imaginary. From general operator-theoretical considerations it may be deduced from the boundedness of $\partial \mathcal{A}$, that the eigenvalues $\zeta$ are discrete.

An example that illustrates this behaviour explicitly is the circular duct $R=1$, where

$$
\phi_{0}=r^{m} \mathrm{e}^{ \pm \mathrm{i} m \theta}, \text { with } \zeta=m \geqslant 0 .
$$

As the shape of the cross section $\mathcal{A}(X)$ changes with $X$, the values of $\zeta$ that allow a solution also change with $X$, and in general there are no solutions possible along the duct for a fixed, given $\zeta$. 
This is of course not true for a duct of constant cross section, and we will show here how in this case the low-frequency solution can be found. Note that this solution is just the unattenuated surface wave, considered in Rienstra[17].

As we saw, $\zeta$ cannot be prescribed because it is an eigenvalue, and therefore essentially part of the solution that depends on $\varepsilon$. So we have to write $\zeta(\varepsilon)$ and expand

$$
\zeta(\varepsilon)=\zeta_{0}+\varepsilon \zeta_{1}+\varepsilon^{2} \zeta_{2}+\ldots
$$

As before in equation (72), we have to leading order the eigenvalue problem

$$
\nabla_{\perp}^{2} \phi_{0}=0, \text { with } \nabla_{\perp} \phi_{0} \cdot \boldsymbol{n}_{\perp}=\zeta_{0} \phi_{0},
$$

with the pair $\left(\phi_{0}, \zeta_{0}\right)$ as the solution. Then, to first order, we have

$$
\nabla_{\perp}^{2} \phi_{1}=0, \text { with } \nabla_{\perp} \phi_{1} \cdot \boldsymbol{n}_{\perp}=\zeta_{0} \phi_{1}+\zeta_{1} \phi_{0}
$$

By applying Green's 2nd identity to $\phi_{1}$ and $\phi_{0}$ we get

$$
\iint_{\mathcal{A}} \phi_{0} \nabla_{\perp}^{2} \phi_{1}-\phi_{1} \nabla_{\perp}^{2} \phi_{0} \mathrm{~d} \sigma=\zeta_{1} \int_{\partial \mathcal{A}} \phi_{0}^{2} \mathrm{~d} \ell=0 .
$$

So $\zeta_{1}=0$ and $\phi_{1} \propto \phi_{0}$. To second order we have (note that now $R_{X}=0$ )

$$
\nabla_{\perp}^{2} \phi_{2}+\kappa^{2} \phi_{0}=0, \quad \text { with } \nabla_{\perp} \phi_{2} \cdot \boldsymbol{n}_{\perp}=\zeta_{0} \phi_{2}+\zeta_{2} \phi_{0} .
$$

Similar to above, we find the solvability condition to be

$$
\iint_{\mathcal{A}} \phi_{0} \nabla_{\perp}^{2} \phi_{2}-\phi_{2} \nabla_{\perp}^{2} \phi_{0} \mathrm{~d} \sigma+\kappa^{2} \iint_{\mathcal{A}} \phi_{0}^{2} \mathrm{~d} \sigma=\zeta_{2} \int_{\partial \mathcal{A}} \phi_{0}^{2} \mathrm{~d} \ell+\kappa^{2} \iint_{\mathcal{A}} \phi_{0}^{2} \mathrm{~d} \sigma=0
$$

yielding $\zeta_{2}$ expressed in $\kappa^{2}$ and integrals of $\phi_{0}^{2}$.

\section{Variable mean soundspeed and density}

If soundspeed $C=C(X, r, \theta)$ and mean density $D=D(X, r, \theta)$ are not uniformly constant, but vary in $r$, $\theta$ and slowly in $x$, we have the reduced wave equation (5), rewritten in slowly varying coordinates,

$$
\varepsilon^{2} \frac{\partial}{\partial X}\left(C^{2} p_{X}\right)+\nabla_{\perp} \cdot\left(C^{2} \nabla_{\perp} p\right)+\varepsilon^{2} \Omega^{2} p=0,
$$

where the dimensionless frequency $\omega=\varepsilon \Omega$ is small. The hard-wall boundary condition is the same as equation (14). When we expand $p=p_{0}+\varepsilon p_{1}+\varepsilon^{2} p_{2} \ldots$, we get to leading order

$$
\nabla_{\perp} \cdot\left(C^{2} \nabla_{\perp} p_{0}\right)=0, \text { with } \nabla_{\perp} p_{0} \cdot \boldsymbol{n}_{\perp}=0,
$$

which has a constant as the solution, so $p_{0}=p_{0}(X)$, a function to be determined. We can derive the same equation for $p_{1}$, to get the same result $\phi_{1}=\phi_{1}(X)$. For the second order we have

$$
\nabla_{\perp} \cdot\left(C^{2} \nabla_{\perp} p_{2}\right)+\frac{\partial}{\partial X}\left(C^{2} p_{0, X}\right)+\Omega^{2} p_{0}=0, \text { with } \nabla_{\perp} p_{2} \cdot \boldsymbol{n}_{\perp}=p_{0, X} \frac{R R_{X}}{\sqrt{R^{2}+R_{\theta}^{2}}} .
$$

We go on to find a solvability condition for $p_{2}$ by integrating this equation along a cross section $\mathcal{A}$. Utilizing the following identity for any differentiable function $f$

$$
\frac{\mathrm{d}}{\mathrm{d} X} \iint_{\mathcal{A}} f(\boldsymbol{X}) \mathrm{d} \sigma=\frac{\mathrm{d}}{\mathrm{d} X} \int_{0}^{2 \pi} \int_{0}^{R} f(X, r, \theta) r \mathrm{~d} r \theta=\int_{0}^{2 \pi} \int_{0}^{R} f_{X} r \mathrm{~d} r \mathrm{~d} \theta+\int_{0}^{2 \pi} f(X, R, \theta) R R_{X} \mathrm{~d} \theta,
$$


we have

$$
\iint_{\mathcal{A}} \nabla_{\perp} \cdot\left(C^{2} \nabla_{\perp} p_{2}\right) \mathrm{d} \sigma=p_{0, X} \int_{0}^{2 \pi} C^{2} R R_{X} \mathrm{~d} \theta=p_{0, X}\left[\frac{\mathrm{d}}{\mathrm{d} X} \iint_{\mathcal{A}} C^{2} \mathrm{~d} \sigma-\iint_{\mathcal{A}} \frac{\partial}{\partial X} C^{2} \mathrm{~d} \sigma\right] .
$$

Furthermore, we have

$$
\iint_{\mathcal{A}} \frac{\partial}{\partial X}\left(C^{2} p_{0, X}\right) \mathrm{d} \sigma=p_{0, X} \iint_{\mathcal{A}} \frac{\partial}{\partial X} C^{2} \mathrm{~d} \sigma+p_{0, X X} \iint_{\mathcal{A}} C^{2} \mathrm{~d} \sigma, \text { and } \iint_{\mathcal{A}} \Omega^{2} p_{0} \mathrm{~d} \sigma=\Omega^{2} p_{0} A .
$$

Then, after introducing the cross-sectional averaged squared sound speed

$$
\overline{C^{2}}=\frac{1}{A} \iint_{\mathcal{A}} C^{2} \mathrm{~d} \sigma
$$

a generalisation of Webster's equation is obtained

$$
A^{-1}\left(A \overline{C^{2}} p_{0, X}\right)_{X}+\Omega^{2} p_{0}=0 .
$$

This may be further simplified by the transformation

$$
A(X) \overline{C^{2}}(X)=d(X)^{2}, \quad p_{0}=d^{-1} \psi
$$

into

$$
\psi^{\prime \prime}+\left(\frac{\Omega^{2}}{\overline{C^{2}}}-\frac{d^{\prime \prime}}{d}\right) \psi=0
$$

\section{Irrotational and isentropic mean flow}

To analyse asymptotically low frequency acoustic perturbations in a slowly varying duct with an irrotational isentropic mean flow, as described by equations (7) and (9), we need to approximate both mean flow and acoustic field to the same order of accuracy.

We start here with the mean flow. In the dimensionless variables used, we have $C^{2}=D^{\gamma-1}$, so equations (7) simplify to

$$
\frac{1}{2} V^{2}+\frac{D^{\gamma-1}}{\gamma-1}=E, \quad \nabla \cdot(D \boldsymbol{V})=0
$$

The mass flux at any cross section $A$ is given by

$$
\iint_{\mathcal{A}} D U \mathrm{~d} \sigma=\mathcal{F} .
$$

Due to the non-dimensionalisation, $U, D, A, \mathcal{F}$ and $E$ are $\mathcal{O}(1)$. Introduce the slow variable $X=\varepsilon x$, and assume $\boldsymbol{V}$ and $D$ to depend essentially on $X$, rather than $x$. We write the velocity as

$$
\boldsymbol{V}=U \boldsymbol{e}_{x}+\boldsymbol{V}_{\perp}
$$

to distinguish between axial and cross-wise components. If flux $\mathcal{F}$ and thermodynamical constant $E$ are independent of $\varepsilon$, we can expand $U=U_{0}+\mathcal{O}\left(\varepsilon^{2}\right)$ and $D=D_{0}+\mathcal{O}\left(\varepsilon^{2}\right)$. As the flow is a potential flow, we can derive, in the same way as in Rienstra[1], that $D_{0}=D_{0}(X), U_{0}=U_{0}(X)$ and $\boldsymbol{V}_{\perp}=\mathcal{O}(\varepsilon)$, satisfying the equations (to be solved numerically)

$$
D_{0} U_{0} A=\mathcal{F}, \quad \frac{\mathcal{F}^{2}}{2 D_{0}^{2} A^{2}}+\frac{D_{0}^{\gamma-1}}{\gamma-1}=E .
$$

We write $\boldsymbol{V}_{\perp}=\varepsilon \widetilde{\boldsymbol{V}}_{\perp}$. 
Next we consider the acoustic field. Using the above results for the mean flow, equation (9) becomes

$$
\nabla_{\perp}^{2} \phi+\varepsilon^{2} D_{0}^{-1}\left(D_{0} \phi_{X}\right)_{X}=\varepsilon^{2}\left(\mathrm{i} \Omega+U_{0} \frac{\partial}{\partial X}+\tilde{\boldsymbol{V}}_{\perp} \cdot \nabla_{\perp}\right)\left[C_{0}^{-2}\left(\mathrm{i} \Omega+U_{0} \frac{\partial}{\partial X}+\widetilde{\boldsymbol{V}}_{\perp} \cdot \nabla_{\perp}\right) \phi\right]
$$

with hard wall boundary condition

$$
\nabla \phi \cdot \boldsymbol{n}=0 \text { at } r=R .
$$

We expand $\phi=\phi_{0}+\varepsilon \phi_{1}+\varepsilon^{2} \phi_{2}+\ldots$ To leading order we have

$$
\nabla_{\perp}^{2} \phi_{0}=0, \quad \nabla_{\perp} \phi_{0} \cdot \boldsymbol{n}_{\perp}=0
$$

yielding the constant solution, i.e., $\phi_{0}=\phi_{0}(X)$.

To first order we have the same equation. To second order we have

$$
\nabla_{\perp}^{2} \phi_{2}+D_{0}^{-1}\left(D_{0} \phi_{0, X}\right)_{X}=\left(\mathrm{i} \Omega+U_{0} \frac{\partial}{\partial X}+\widetilde{\boldsymbol{V}}_{\perp} \cdot \nabla_{\perp}\right)\left[C_{0}^{-2}\left(\mathrm{i} \Omega+U_{0} \frac{\partial}{\partial X}+\widetilde{\boldsymbol{V}}_{\perp} \cdot \nabla_{\perp}\right) \phi_{0}\right]
$$

with boundary conditions given by equation (19). After integration across a cross section $\mathcal{A}(X)$, we obtain, similar to before, the Webster equation generalised for irrotational isentropic mean flow

$$
\left(D_{0} A\right)^{-1}\left(D_{0} A \phi_{0, X}\right)_{X}=\left(\mathrm{i} \Omega+U_{0} \frac{\partial}{\partial X}\right)\left[C_{0}^{-2}\left(\mathrm{i} \Omega+U_{0} \frac{\partial}{\partial X}\right) \phi_{0}\right] .
$$

Note that this equation seems to be equivalent to the set given by Pierce[13, p. 422] apart from a factor $\frac{1}{2}$.

\subsection{Mean flow with impedance walls.}

The problem with mean flow and an impedance wall is more intricate. Instead of the duct wall boundary condition given in equation (68), we have Myers' condition[18], rewritten according to Eversman[19], as follows

$$
\mathrm{i} \omega D(\boldsymbol{v} \cdot \boldsymbol{n})=\frac{\mathrm{i} \omega D p}{Z}+\frac{\mathrm{d}}{\mathrm{d} \tau}\left(\frac{D V_{\tau} p}{Z}\right)
$$

$V_{\tau}$ denotes the component of the mean flow vector tangential to the duct wall and $\tau$ denotes the tangential coordinate in that direction. The following identity, obtained from the path of a particle following a streamline along the wall,

$$
\frac{\mathrm{d}}{\mathrm{d} \tau}=\frac{\mathrm{d} t}{\mathrm{~d} \tau} \frac{\mathrm{d} x}{\mathrm{~d} t} \frac{\mathrm{d}}{\mathrm{d} x}=\varepsilon \frac{U}{V_{\tau}} \frac{\mathrm{d}}{\mathrm{d} X}
$$

can be used to further simplify the boundary condition (we introduce our notation)

$$
\mathrm{i} \Omega D(\nabla \phi \cdot \boldsymbol{n})=\frac{1}{V_{\tau}}\left(\mathrm{i} \Omega+U \frac{\mathrm{d}}{\mathrm{d} X}\right)\left(\frac{D V_{\tau} p}{Z}\right) .
$$

We note that $p=\mathcal{O}(\varepsilon)$ if $\phi=\mathcal{O}(1)$ since along the wall

$$
p=-\varepsilon D\left(\mathrm{i} \Omega+U \frac{\mathrm{d}}{\mathrm{d} X}\right) \phi .
$$

If we consider the case $Z=\mathcal{O}(1)$, and expand $\phi=\phi_{0}+\varepsilon \phi_{1}+\ldots$, we get to leading order, as before,

$$
\nabla_{\perp}^{2} \phi_{0}=0, \text { with } \nabla_{\perp} \phi_{0} \cdot \boldsymbol{n}_{\perp}=0,
$$

so $\phi_{0}=\phi_{0}(X)$. To first order we have the same equation $\nabla_{\perp}^{2} \phi_{1}=0$ for $\phi_{1}$. With $V_{\tau}=U_{0}+\mathcal{O}\left(\varepsilon^{2}\right)$, the boundary condition is now

$$
\mathrm{i} \Omega D_{0} U_{0}\left(\nabla_{\perp} \phi_{1} \cdot \boldsymbol{n}_{\perp}\right)=\left(\mathrm{i} \Omega+U_{0} \frac{\mathrm{d}}{\mathrm{d} X}\right)\left(\frac{D_{0} U_{0} p_{0}}{Z}\right) .
$$

From

$$
\iint_{\mathcal{A}} \nabla_{\perp}^{2} \phi_{1} \mathrm{~d} \sigma=\int_{\partial \mathcal{A}} \nabla_{\perp} \phi_{1} \cdot \boldsymbol{n}_{\perp} \mathrm{d} \ell=0
$$

together with equation (100), it follows that possible solutions are given by $p_{0}(X) \equiv 0$, i.e., purely convected perturbations. 


\section{Conclusions}

Webster's classic horn equation and some generalisations have been (re)derived systematically, as an asymptotic perturbation problem, from a number of modelling assumptions by the method of slow variation. The conditions on frequency, medium and geometry are explicitly indicated. The error and higher order corrections are also explicitly stated. The presence of lining is shown to allow in general only the trivial solution. A curved duct is shown to produce the same equation if the radius of curvature is not smaller than the typical wave length or duct length scale. The approximation is non-uniform near source or entrance. The prevailing boundary layer solution for an arbitrary duct cross section is given.

\section{Acknowledgement}

This work was carried out in the context of the "TurboNoiseCFD" project of the European Union's 5th Framework "Growth" Programme.

We gratefully acknowledge the cooperation with J. de Graaf and T.D. Chandra on the analysis of the entrance boundary layer. We thank N.C. Ovenden for helpfull discussions and critical reading of the text.

\section{References}

[1] S.W. Rienstra, 1999, Sound Transmission in Slowly Varying Circular and Annular Ducts with Flow, Journal of Fluid Mechanics 380, 279-296.

[2] S.W. Rienstra and W. Eversman, 2001, A Numerical Comparison Between Multiple-Scales and FEM Solution for Sound Propagation in Lined Flow Ducts, Journal of Fluid Mechanics 437, 367 - 384.

[3] M. VAn Dyke, Slow Variations in Continuum Mechanics, in: Advances in Applied Mechanics, Volume 25, pp. 1-45, Academic Press, Orlando, 1987.

[4] P.A. Lagerstrom, Matched Asymptotic Expansions: Ideas and Techniques, Springer-Verlag, New York, 1988

[5] W. Eckhaus, Asymptotic Analysis of Singular Perturbations, North Holland, Amsterdam, 1979.

[6] A.H. NAYFEH, Perturbation Methods, John Wiley \& Sons, Inc., New York, 1973

[7] A.G. Webster. 1919, Acoustical impedance, and the theory of horns and of the phonograph, Proc. Natl. Acad. Sci. (U.S.) 5, 275-282. reprinted in: Journal of Audio Engineering Society, 1977, 25(1-2), 24-28

[8] P.M. Morse. Vibration and sound. McGraw-Hill, New York, 2nd edition, 1948.

[9] V. SAlmon, 1946, Generalized plane wave horn theory, Journal of the Acoustical Society of America 17, 199-218.

[10] V. SAlmon, 1946, A new family of horns, Journal of the Acoustical Society of America 17, 212-218.

[11] E. EISNER, 1967, Complete Solutions of the Webster Horn Equation, Journal of the Acoustical Society of America $41,1126$.

[12] A.H. Benade and E.V. Jansson, 1974, On Plane and Spherical Waves in Horns with Nonuniform Flare. I. Theory of Radiation, Resonance Frequencies, and Mode Conversion, Acustica 31(2), 79-98.

[13] A.D. PIERCE. Acoustics: an Introduction to its Physical Principles and Applications. McGraw-Hill Book Company, Inc., New York, 1981. Also available from the Acoustical Society of America.

[14] T.D. Chandra, Perturbation and Operator Methods for Solving Stokes Flow and Heat Flow Problems, PhD Thesis, Eindhoven: Technische Universiteit Eindhoven, May 2002, ISBN 90-386-0542-0

[15] I.S. Gradshteyn and I.M. Ryzhik, I.M., Table of Integrals, Series and Products, edited by Alan Jeffrey, 5th edition, Academic Press, London, 1994

[16] M.M.Lipschutz, Differential Geometry, Schaum's Outline Series, McGraw-Hill Book Company, New York, 1969.

[17] S.W. RIENStra, 2001, A Classification of Duct Modes Based on Surface Waves, AIAA 2001-2180, 7th AIAA/CEAS Aeroacoustics Conference in Maastricht, The Netherlands, 28-30 May 2001.

[18] M.K. Myers, 1980, On the Acoustic Boundary Condition in the Presence of Flow. Journal of Sound and Vibration 71(3), $429-434$.

[19] W. Eversman, 2001, The Boundary Condition at an Impedance Wall in a Non-Uniform Duct with Potential Mean Flow, Journal of Sound and Vibration 246(1), 63-69. 